\title{
Discovery of eclipsing binary central stars in the planetary nebulae M 3-16, H 2-29, and M 2-19
}

\author{
B. Miszalski ${ }^{1,2}$, A. Acker ${ }^{1}$, A. F. J. Moffat ${ }^{3}$, Q. A. Parker ${ }^{2,4}$, and A. Udalski ${ }^{5}$ \\ 1 Observatoire Astronomique, Université Louis Pasteur, 67000 Strasbourg, France \\ e-mail: brent@newb6.u-strasbg.fr, acker@newb6.u-strasbg.fr \\ 2 Department of Physics, Macquarie University, Sydney NSW 2109, Australia \\ e-mail: qap@ics.mq.edu.au \\ 3 Dépt. de physique, Univ. de Montréal CP 6128, Succ. Centre-Ville, Montréal, QC H3C 3J7, Canada \\ e-mail: moffat@astro.umontreal.ca \\ 4 Anglo-Australian Observatory, Epping, NSW 1710, Australia \\ 5 Warsaw University Observatory, Al. Ujazdowskie 4, 00-478 Warsaw, Poland \\ e-mail: udalski@astrouw.edu.pl
}

Received 5 July 2008 / Accepted 4 August 2008

\section{ABSTRACT}

\begin{abstract}
Progress in understanding the formation and evolution of planetary nebulae (PN) has been restricted by a paucity of well-determined central star masses. To address this deficiency we aim to (i) increase significantly the number of known eclipsing binary central stars of PN (CSPN); and subsequently (ii) measure directly their masses and absolute dimensions by combining their light curve parameters with planned radial velocity data. Using photometric data from the third phase of the Optical Gravitational Lensing Experiment (OGLE), we searched for periodic variability in a large sample of PN towards the Galactic Bulge using Fourier and phase-dispersion minimisation techniques. Among some dozen periodically variable CSPN found, we report on three new eclipsing binaries: M 3-16, H 2-29 and M 2-19. We present images, confirmatory spectroscopy and light curves of the systems.
\end{abstract}

Key words. stars: binaries: eclipsing - ISM: planetary nebulae: general

\section{Introduction}

The key parameter for any star is its mass. The least modeldependent method to obtain masses is by means of Keplerian orbits in binary systems. Eclipsing binaries provide critical information necessary to secure the masses and other fundamental stellar parameters. The mass is determined from radial velocity (RV) orbits of both stars and the orbital inclination deduced from photometric eclipses.

Directly measured masses have not been fully exploited in the case of central stars of planetary nebulae (CSPN), partly because so few eclipsing binary systems are known. Indirect methods, such as nebula modelling (e.g. Gesicki et al. 2006) or model atmosphere fitting (e.g. Napiwotzki 1999), offer a poor alternative to relatively model-independent techniques such as Keplerian orbits.

Obtaining direct masses for a sufficiently large number of CSPN is key to understanding the link between the AGB phase of stellar evolution and the final white dwarf stage. Such a study will also significantly improve our understanding of PN shaping mechanisms (e.g. Zijlstra 2007) and can provide compelling candidates for SN Ia progenitors (Tovmassian et al. 2008). Because most stars of low-intermediate mass experience the PN stage, PN are also major contributors to ISM enrichment and the chemical evolution of galaxies.

De Marco et al. (2008, and references therein) summarised the status of photometrically variable CSPN, including 12 close binaries with known periods. Three of these are eclipsing: UU Sge (Pollaco \& Bell 1993), V477 Lyr (Pollaco \& Bell 1994), and BE UMa (Ferguson et al. 1999). All three have viable mass determinations using both RV and light curves, with $0.5-0.7 M_{\odot}$ for the hot $60-120 \mathrm{kK}$ primary and $0.15-0.36 M_{\odot}$ for the irradiated $5-7 \mathrm{kK}$ secondary. Their light curves display strong irradiation effects, in addition to typical emission lines from the heated hemisphere of the secondary. All these systems are believed to be post-CE (common-envelope) binaries, where the secondary spirals in through the primary's AGB envelope. The remaining stars of De Marco et al. (2008) are non-eclipsing and therefore any mass estimates for them are subject to considerable uncertainty.

Either RV or photometric monitoring surveys may be used to find CSPN binaries. However, if the ultimate goal (as in this work) is to determine the masses of the binaries, then it is easier and more efficient to start with photometric surveys to preselect eclipsing binaries, for which RV orbits combined with photometrically-derived inclinations secure the masses. Indeed, RV monitoring surveys have proven to be difficult with many candidates showing RV variability without definitive periodicity (e.g. De Marco et al. 2004).

This paper introduces a novel use of extant online photometric data from the OGLE microlensing survey. In Sect. 2 we describe a search for periodic variability in over $300 \mathrm{PN}$ towards the Galactic Bulge. In Sect. 3 we present the discovery of three new eclipsing binary CSPN. We discuss the nature of these new discoveries in Sect. 4 and conclude in Sect. 5. 


\section{CSPN variability towards the Galactic Bulge}

\subsection{OGLE-III}

To address the paucity of binary CSPN we have searched for periodic photometric variations in data from the Optical Gravitational Lensing Experiment (OGLE; Udalski et al. 1992) in its third phase (OGLE-III; e.g. Udalski et al. 2002). Each phase of the OGLE project improves its temporal and areal coverage of the Galactic Bulge and Magellanic Clouds over previous phases. The areal coverage of OGLE-III is very wellmatched to the PN distribution towards the Bulge (e.g. the most frequently sampled fields cover $|\ell| \leq 5$ and $-5<b<-2$ ).

The OGLE-III survey uses the $1.3-\mathrm{m}$ Warsaw telescope at the Las Campanas Observatory, Chile. It is equipped with a $\mathrm{CCD}$ mosaic camera with eight $2 \mathrm{~K} \times 4 \mathrm{~K}$ CCDs providing a $35 \times 35.5 \operatorname{arcmin}^{2}$ field of view with $0.26^{\prime \prime}$ pixels. The survey is conducted in the $I$-band reaching a limiting magnitude of $I \sim 20$, although some $V$-band reference images are also available. The data span approximately six years starting from June 2001 with gaps between each Bulge season.

\subsection{Search method}

Surveys for PN towards the Galactic Bulge have been enhanced considerably by the Macquarie/AAO/Strasbourg $\mathrm{H} \alpha$ PN catalogues (MASH; Parker et al. 2006; Miszalski et al. 2008). By effectively doubling the PN population towards the Bulge, MASH now enables a large population of Galactic PN to be monitored for variability in a homogeneous fashion by one photometric survey.

For each of the over $300 \mathrm{PN}$ that were located in the OGLEIII survey fields, we examined OGLE-III $I$-band finder charts to identify central stars for which time-series photometry could be extracted. Approximately $30 \%$ of these PN had CSPN that were identified unambiguously with the help of significantly lower nebular contamination in the $I$-band. In more difficult cases we selected a number of likely candidates close to the geometric centre of each PN.

Time series photometry for each identified CSPN was subsequently retrieved from the OGLE-III database generated by a data reduction pipeline based on the difference image analysis (DIA) method (Woźniak 2000). The stellar-optimised pipeline could not extract some light curves in cases of nebular contamination. We examined the photometric data using both the Fourier analysis package PERIOD04 (Lenz \& Breger 2004) and the phase dispersion minimisation (PDM) IRAF task (Stellingwerf 1978). Initially, the data were searched with the Fourier technique for frequencies in the range $0-50 \mathrm{~d}^{-1}$, the latter being the typical Nyquist frequency for well-sampled fields, with a step rate $2.2 \times 10^{-4} \mathrm{~d}^{-1}(\sim 1 / 5$ times the inverse sample length of $\sim 6$ years). Periodicity was identified when the Fourier peak amplitude reached at least $3 \sigma$ above the noise. The final selection of the most likely frequency was made after visual inspection of the phased light curve. We used the PDM technique to check our Fourier results for $P \sim 0.01-4$ days.

\section{Results}

Some dozen periodically variable CSPN were found during our examination of OGLE-III data. A photometric binary fraction of 10-15\% (Bond 2000) compares reasonably well with our results, but complex selection criteria involving CSPN identification and uneven field sampling precludes a meaningful estimate of our binary fraction from being given here. A future paper will be dedicated to this topic (Miszalski et al., in prep).

\subsection{Three new eclipsing binary CSPN}

Of all the periodically variable CSPN found, those of M 3-16 (PN G359.1-02.3), H 2-29 (PN G357.6-03.3) and M 2-19 (PN G000.2-01.9) have distinctive eclipsing light curves. These PN have been known for some time (Acker et al. 1992) but are all relatively unstudied (although see Sect. 3.3). The other variables, including some MASH CSPN, tend to exhibit sinusoidallike light curves and will be discussed elsewhere. In Fig. 1, for each object we present an $\mathrm{H} \alpha$ image, the OGLE-III I-band image and its OGLE-III light curve. The data are described in the following sections.

\subsection{Photometry}

\subsection{1. $\mathrm{H} \alpha$ images}

High-quality $\mathrm{H} \alpha$ images of each nebula are presented in the first column of Fig. 1. We obtained an $\mathrm{H} \alpha$ image of M 3-16 using the Mosaic II camera on the CTIO 4-m Blanco telescope in June 2008. The H 2-29 and M 2-19 images were taken with ESO NTT/EMMI during programs 67.D-0527(A) and 71.D-0448(A) (Ruffle et al. 2004). We note that all images include both adjacent $[\mathrm{NII}]$ emission lines in the bandpass of the $\mathrm{H} \alpha$ filters. In all instances the resolution is well-matched to the OGLE-III resolution of $0.26^{\prime \prime} /$ pixel.

\subsubsection{CSPN identification}

Identification of central stars in the OGLE-III I-band images requires careful scrutiny and a full discussion of selection effects will be given in Miszalski et al. (in prep.). Nevertheless, in each of our three eclipsing binary CSPN we have unambiguously identified the CSPN, as indicated by the markers in Fig. 1. In the case of M 3-16 and M 2-19 we detect faint nebulae on the $I$-band images with centrally located stars. Although no $I$-band nebular emission can be seen for H 2-29, the star marked is centrally located with respect to the nebula in the $\mathrm{H} \alpha$ image. Furthermore, we note that the CSPN marked is brighter in the OGLE-III $V$ than the $I$-band image, which suggests strongly that the star is blue and is therefore the actual CSPN.

\subsubsection{Light curves}

Phased light curves of each CSPN are given in Fig. 1, with periods and ephemerides given in Table 1 . No significant change in period was detected between each individual Bulge season for each object.

We visually inspected the light curves phased with the period recovered by the Fourier method. We doubled the period of M 3-16 to separate the overlapping, unequal eclipses. The slightly rounded shape of its light curve between minima is interpreted as ellipsoidal variations. The light curve of $\mathrm{H} \mathrm{2-29}$ phased with the initial Fourier period shows a moderately weak secondary minimum at 0.5 phase leading us to accept it as the most likely period. However, if the secondary minimum is spurious, then the true period would likely be twice our value. When phased with the Fourier period, the light curve of M 2-19 lacks a convincing secondary minimum at phase 0.5 and shows an unusually wide primary minimum. Therefore, we doubled the Fourier period of M 2-19. 


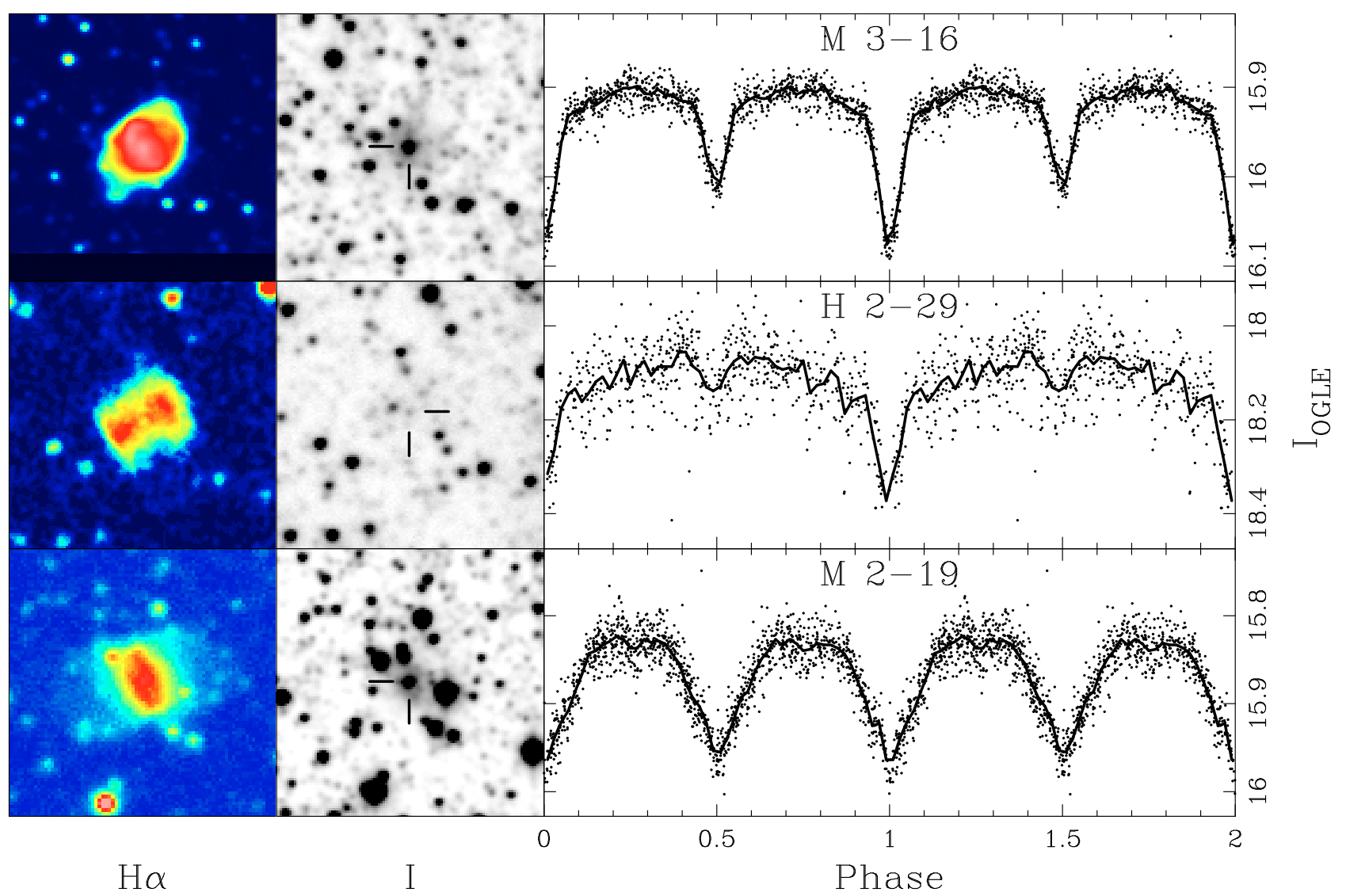

Fig. 1. A montage of the three newly discovered eclipsing systems. Each row depicts from left to right: an H $\alpha$ image of the nebula, an OGLE-III $I$-band image, and a phased OGLE-III light curve of the central star (marked on the $I$-band image). Periods and ephemerides are given in Table 1. All images are $30 \times 30 \operatorname{arcsec}^{2}$ with North to top and East to left. The solid curve is a binned light curve representation sampled at $\delta \phi=0.01$.

Table 1. Basic data for the three new eclipsing binaries. An average $I$-band magnitude $\bar{m}_{I}$ is calculated from the OGLE-III time-series photometry of $N$ observations over six Galactic Bulge seasons. The epoch of primary minimum $E_{0}$ is also given. The current zeropoint of $\bar{m}_{I}$ is roughly calibrated to an uncertainty of $\sim 0.1-0.2 \mathrm{mag}$.

\begin{tabular}{lllllllll}
\hline \hline PN G & Name & RA (J2000) & Dec (J2000) & OGLE field & $E_{0}$ (HJD-2 450 000) & $P$ (days) & $\bar{m}_{I}$ & $N$ \\
\hline $359.1-02.3$ & M 3-16 & 175246.1 & -304935 & BLG180.1 & $3850.9184 \pm 0.0015$ & $0.573648 \pm 0.000020$ & 15.93 & 1039 \\
$357.6-03.3$ & H 2-29 & 175316.8 & -324038 & BLG155.1 & $3619.5208 \pm 0.0025$ & $0.244110 \pm 0.000005$ & 18.12 & 609 \\
$000.2-01.9$ & M 2-19 & 175345.6 & -294346 & BLG101.3 & $4224.7822 \pm 0.0035$ & $0.670170 \pm 0.000020$ & 15.87 & 1273 \\
\hline
\end{tabular}

\subsection{Spectroscopy}

Although nebular spectroscopy exists for all our objects, no identification of CSPN features has been reported in the literature (Cuisinier et al. 2000; Exter et al. 2004). Spectroscopic observations of M 3-16 and M 2-19 were made with the AAOmega system on the 3.9-m Anglo-Australian Telescope (AAT), on the nights of 16 March 2008 and 29 May 2008. AAOmega is an optical multi-object spectrograph (Sharp et al. 2006) that is fibre-fed by the $2 \mathrm{dF}$ fibre positioner (Lewis et al. 2002). The data were taken in service mode and exposure times were $2 \times 1500 \mathrm{~s}$ for both M 3-16 and M 2-19 with an additional 1800 s on M 2-19. We used the $580 \mathrm{~V}$ and $385 \mathrm{R}$ volume-phase holographic (VPH) gratings for the blue and red arms, respectively. This provided a central resolution of $3.5 \AA$ in the blue and $5.3 \AA$ in the red and a wavelength coverage of 3700-5800 $\AA$ and 5720-8850 A, respectively.

The two epochs for M 3-16 and three epochs for M 2-19 were summed to give a $\mathrm{S} / \mathrm{N}$ in the continuum at $5300 \AA$ of $\sim 40$ and $\sim 30$, respectively. Both spectra exhibit stellar HeII absorption lines as expected for hot O-type central stars (Fig. 2). Evidence for stellar lines that betray a cool secondary component is weak. The CaII triplet feature is not seen and the possible presence of very faint N III and C III emission lines at $\sim 4640 \AA$ requires confirmation (see Pollacco \& Bell 1993, 1994). These features are more likely to be present in H 2-29, but no sufficiently deep spectra currently exist. Our confirmatory spectra of low S/N (individually) and low resolution were not intended to uncover radial velocity variations. Nevertheless, despite large uncertainties, we noticed no variation in the radial velocity of the He II lines with respect to nebular lines. Higher resolution data is required to place limits on any possible variation.

\section{Discussion}

\subsection{Nature of the systems}

At this stage we can only comment on the components of each of our new systems and leave analysis using the Wilson-Devinney method (Wilson \& Devinney 1971) to future papers. The periods of our systems are short and are within the range of the periods known for the majority of binary CSPN (De Marco et al. 2008). 


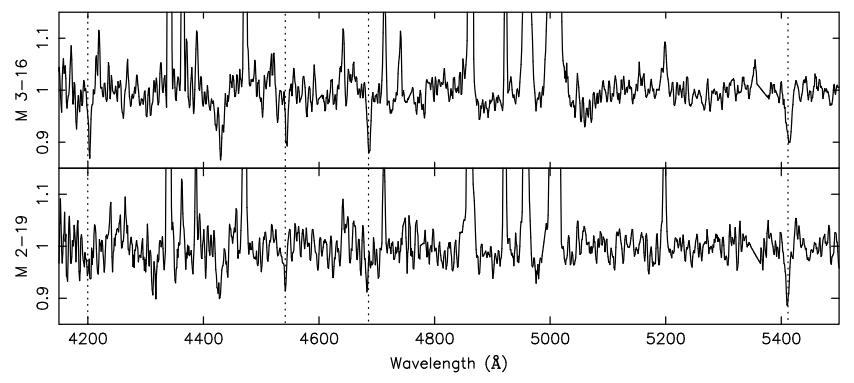

Fig. 2. AAOmega spectroscopy for M 3-16 (top) and M 2-19 (bottom) showing stellar He II absorption lines (dotted lines). Contaminating nebular emission lines are also present. The spectra have been rectified and smoothed.

Our light curves show none of the very strong irradiation effects seen for UU Sge, V477 Lyr or BE UMa, suggesting a very different system composition, although irradiation effects may be present in H 2-29, albeit weaker and uncertain. For M 2-19 and M 3-16 in particular, the eclipses are of similar and modest depths suggesting comparable effective temperatures of both components.

Our low-resolution confirmatory spectroscopy show no detectable difference between radial velocities of He II absorption lines and nebular lines in M 2-19 and M 3-16. This is most likely due to our limited sensitivity to radial velocity variations of up to $50-150 \mathrm{~km} \mathrm{~s}^{-1}$ observed in known eclipsing CSPN (Pollacco \& Bell 1993, 1994). If these large variations are not confirmed by future monitoring, then we may be seeing the hot, ionising component of a triple system with eclipses generated by two mainsequence stars of similar effective temperatures. This scenario is supported by the lack of strong irradiation effects and the similar eclipse depth in the light curves of M 2-19 and M 3-16. However, we do not see any convincing stellar features indicative of this scenario in our low $\mathrm{S} / \mathrm{N}$ spectra. Such a case was suggested for SuWt 2 that has a similar light curve to M 3-16, but its bright A-type double-lined spectroscopic and eclipsing binary CSPN has not been excluded as a foreground object (Bond et al. 2000). There may be another explanation and more radial velocity data at higher resolution are needed to resolve the issue.

\subsection{Morphology}

The morphologies of our three nebulae all show bipolar features. This is consistent with the tendency for close binary stars to eject non-spherical nebulae through interacting stellar winds and tidal effects (e.g. Soker 1997). The morphology of H 2-29 was indeed considered to be a likely product of CE evolution with a stellar companion (Soker 1997).

The "butterfly" shape has been proposed as the preferred morphology of nebulae ejected by close binaries because it is the most efficient transfer mechanism of angular momentum to the ejecta (Zijlstra 2007). M 2-19 exhibits the classical "butterfly" morphology and is the first firmly established case with a considerably short period supporting this hypothesis. Other "butterfly" nebulae with a binary nucleus include NGC 2346 (Mendez et al. 1982), but it has a longer period of 16 days.
M 3-16 exhibits the most complex morphology of the three. Surrounding the south-eastern side of the central star we see what may be part of an equatorial torus-like structure. Perpendicular to this we have two arms often seen in other bipolar nebulae that extend towards the north-west. If we use this to define an axis of symmetry, then 25 degrees away from this axis we have two opposing broad jets or ansae.

\section{Conclusions}

We have doubled the number of eclipsing binary CSPN after searching OGLE-III photometry in a large PN catalogue towards the Galactic Bulge. The post-CE CSPN show bipolar morphologies consistent with the current hypothesis that close binaries lead to non-spherical nebulae. A future paper will present more new close binary CSPN detections, discuss the selection effects of the search in detail, and provide a new, independent estimate of the binary fraction of PN. Planned high-resolution spectroscopy will refine system parameters and derive masses of our new eclipsing binaries.

Acknowledgements. B.M. acknowledges the support of an Australian Postgraduate Award and support from PICS/ULP. Thanks to Paul Dobbie for taking some of the AAOmega service observations and to David Frew for valuable discussions. A.F.J.M. is grateful for financial assistance to NSERC (Canada) and FQRNT (Québec). The OGLE project is partially supported by the Polish MNiSW grant N20303032/4275. We thank the referee for very helpful comments.

\section{References}

Acker, A., Marcout, J., Ochsenbein, F., Stenholm, B., \& Tylenda, R. 1992, Garching: European Southern Observatory

Bond, H. E. 2000, Asymmetrical Planetary Nebulae II: From Origins to Microstructures, 199, 115

Cuisinier, F., Maciel, W. J., Köppen, J., Acker, A., \& Stenholm, B. 2000, A\&A, 353,543

De Marco, O., Bond, H. E., Harmer, D., et al. 2004, ApJ, 602, L93

De Marco, O., Hillwig, T. C., \& Smith, A. J. 2008, AJ, 136, 323

Exter, K. M., Barlow, M. J., \& Walton, N. A. 2004, MNRAS, 349, 1291

Ferguson, D. H., Liebert, J., Haas, S., et al. 1999, ApJ, 518, 866

Gesicki, K., Zijlstra, A. A., Acker, A., et al. 2006, A\&A, 451, 925

Lenz, P., \& Breger, M. 2004, The A-Star Puzzle, 224, 786

Lewis, I. J., Cannon, R. D., Taylor, K., et al. 2002, MNRAS, 333, 279

Mendez, R. H., Gathier, R., \& Niemela, V. S. 1982, A\&A, 116, L5

Miszalski, B., Parker, Q. A., Acker, A., et al. 2008, MNRAS, 384, 525

Napiwotzki, R. 1999, A\&A, 350, 101

Parker, Q. A., Acker, A., Frew, D. J., et al. 2006, MNRAS, 373, 79

Pollacco, D. L., \& Bell, S. A. 1993, MNRAS, 262, 377

Pollacco, D. L., \& Bell, S. A. 1994, MNRAS, 267, 452

Ruffle, P. M. E., Zijlstra, A. A., Walsh, J. R., et al. 2004, MNRAS, 353, 796

Sharp, R., Saunders, W., Smith, G., et al. 2006, SPIE, 6269, 14

Soker, N. 1997, ApJS, 112, 487

Stellingwerf, R. F. 1978, ApJ, 224, 953

Tovmassian, G., Tomsick, J., Napiwotzki, R., et al. 2008, Astrophys. Compact Objects, 968, 62

Udalski, A., Szymanski, M., Kaluzny, J., et al. 1992, Acta Astron., 42, 253

Udalski, A., Paczynski, B., Zebrun, K., et al. 2002, Acta Astron., 52, 1

Wilson, R. E., \& Devinney, E. J. 1971, ApJ, 166, 605

Wozniak, P. R. 2000, Acta Astron., 50, 421

Zijlstra, A. A. 2007, Baltic Astron., 16, 79 